\title{
Longterm Outcome of Patients with Primary Antiphospholipid Syndrome: A Retrospective Multicenter Study
}

\author{
Mara Taraborelli, Rossella Reggia, Francesca Dall'Ara, Micaela Fredi, Laura Andreoli, \\ Maria Gerosa, Ariela Hoxha, Laura Massaro, Marta Tonello, Nathalie Costedoat-Chalumeau, \\ Patrice Cacoub, Franco Franceschini, Pier Luigi Meroni, Jean Charles Piette, Amelia Ruffatti, \\ Guido Valesini, Eon Nigel Harris, and Angela Tincani
}

ABSTRACT. Objective. To assess the longterm frequency of thrombotic recurrences, obstetrical complications, organ damage, severe comorbidities, and evolution toward connective tissue disease (CTD) in primary antiphospholipid syndrome (PAPS).

Methods. Medical records of patients with PAPS followed in 6 centers for $\geq 15$ years were retrospectively reviewed.

Results. One hundred fifteen patients were studied: 88\% women, followed between 1983 and 2014 with a mean $( \pm$ SD) age at diagnosis of $33( \pm 10)$ years. During a median followup of 18 years (range 15-30), 50 patients (44\%) had at least a thrombotic event for a total of 75 events and an annual incidence of $3.5 \%$. Thromboses were more frequent in patients with previous thrombotic history $(\mathrm{p}=0.002)$. A catastrophic antiphospholipid syndrome occurred in 6 patients $(5 \%)$. The use of oral anticoagulants in patients with thrombotic onset did not appear to be protective against recurrences $(p=0.26)$. Fifty-two women had 87 pregnancies, successful in $78 \%$. Twenty-nine percent of patients accrued functional damage. Damage was significantly associated with a thrombotic history $(\mathrm{p}=0.004)$ and with arterial events $(\mathrm{p}<0.001)$, especially stroke, but not with demographics, serology, or treatment. Twenty-four major bleeding episodes were recorded in 18 patients, all receiving anticoagulants. Severe infections affected 6 patients (5\%), with 1 fatality. A solid cancer was diagnosed in 8 patients $(7 \%)$. Altogether, 16 patients $(14 \%)$ developed an autoimmune disease and $13(11 \%)$ a full-blown picture of CTD.

Conclusion. Despite therapy, a high proportion of patients experienced new thrombotic events and organ damage, while evolution toward CTD was infrequent. (First Release June 1 2017; J Rheumatol 2017;44:1165-72; doi:10.3899/jrheum.161364)

Key Indexing Terms:

PRIMARY ANTIPHOSPHOLIPID SYNDROME RECURRENCES PREGNANCY $\begin{array}{lll}\text { EVOLUTION ORGAN DAMAGE THROMBOSIS } & \text { ORON }\end{array}$

From the Rheumatology and Clinical Immunology Department, Spedali Civili and University of Brescia, Brescia; Division of Rheumatology, Department of Clinical Sciences and Community Health, Ospedale Gaetano Pini, University of Milan, Milan; Rheumatology Unit,

Department of Medicine - DIMED, University of Padua, Padua; Internal Medicine and Medical Specialties Department, Policlinico Umberto I, La Sapienza University of Rome, Rome, Italy; AP-HP, Hôpital Cochin, Centre de référence maladies auto-immunes et systémiques rares, Service de Médecine Interne; AP-HP, Hospital Pitié-Salpêtrière, Centre de référence maladies auto-immunes et systémiques rares, Service de Médecine Interne, Pierre et Marie Curie Paris VI University, Paris, France; University of the West Indies, Kingston, Jamaica.

M. Taraborelli, MD, Rheumatology and Clinical Immunology Department, Spedali Civili and University of Brescia; R. Reggia, MD, Rheumatology and Clinical Immunology Department, Spedali Civili and University of Brescia; F. Dall'Ara, MD, Rheumatology and Clinical Immunology Department, Spedali Civili and University of Brescia; M. Fredi, MD, Rheumatology and Clinical Immunology Department, Spedali Civili and University of Brescia; L. Andreoli, MD, PhD, Rheumatology and Clinical Immunology Department, Spedali Civili and University of Brescia;

M. Gerosa, MD, PhD, Division of Rheumatology, Department of Clinical Sciences and Community Health, Ospedale Gaetano Pini, University of Milan; A. Hoxha, MD, PhD, Rheumatology Unit, Department of Medicine - DIMED, University of Padua; L. Massaro, MD, Internal Medicine and Medical Specialties Department, Policlinico Umberto I, La Sapienza

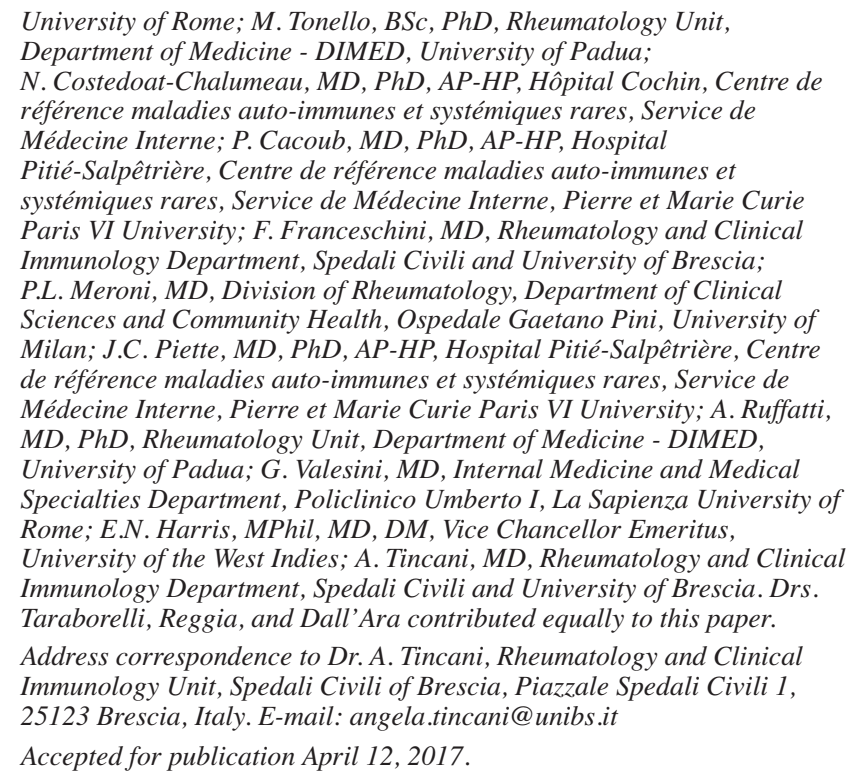
Department of Medicine - DIMED, University of Padua; N. Costedoat-Chalumeau, MD, PhD, AP-HP, Hôpital Cochin, Centre de référence maladies auto-immunes et systémiques rares, Service de Médecine Interne; P. Cacoub, MD, PhD, AP-HP, Hospital Pitié-Salpêtrière, Centre de référence maladies auto-immunes et systémiques rares, Service de Médecine Interne, Pierre et Marie Curie Paris VI University; F. Franceschini, MD, Rheumatology and Clinical Immunology Department, Spedali Civili and University of Brescia; P.L. Meroni, MD, Division of Rheumatology, Department of Clinical Sciences and Community Health, Ospedale Gaetano Pini, University of Milan; J.C. Piette, MD, PhD, AP-HP, Hospital Pitié-Salpêtrière, Centre de référence maladies auto-immunes et systémiques rares, Service de Médecine Interne, Pierre et Marie Curie Paris VI University; A. Ruffatti, $M D, P h D$, Rheumatology Unit, Department of Medicine - DIMED, University of Padua; G. Valesini, MD, Internal Medicine and Medical Specialties Department, Policlinico Umberto I, La Sapienza University of Rome; E.N. Harris, MPhil, MD, DM, Vice Chancellor Emeritus, University of the West Indies; A. Tincani, MD, Rheumatology and Clinical Immunology Department, Spedali Civili and University of Brescia. Drs. Taraborelli, Reggia, and Dall'Ara contributed equally to this paper. Address correspondence to Dr. A. Tincani, Rheumatology and Clinical Immunology Unit, Spedali Civili of Brescia, Piazzale Spedali Civili 1, 25123 Brescia, Italy.E-mail: angela.tincani@unibs.it Accepted for publication April 12, 2017. 
Antiphospholipid syndrome (APS) is an acquired systemic autoimmune disease characterized by the presence of recurrent thrombotic events, obstetrical morbidity, and antiphospholipid antibodies (aPL) ${ }^{1}$. Although acute disease manifestations are well known, information on the longterm outcome is still very limited and most of the data come from mixed cohorts including primary APS (PAPS) and APS associated with other autoimmune diseases ${ }^{2,3}$. To our knowledge, no longterm data are available regarding PAPS.

For these reasons a multicenter study involving different referral centers with consolidated experience on APS was planned to clarify the prognosis of patients with PAPS. We retrospectively analyzed a cohort of patients with PAPS all attending the same tertiary care center for more than 15 years, focusing on the frequency of aPL-related events, the organ damage accrual (with related risk factors), the occurrence of severe comorbidities (i.e., infections, hemorrhages, malignancies), and the possible evolution toward other systemic autoimmune diseases. We were also interested in the changes of serological profile and cardiovascular $(\mathrm{CV})$ risk factors during the observation time.

\section{MATERIALS AND METHODS}

Patients. We retrospectively reviewed medical records of consecutive patients with PAPS, retrospectively classified according to the updated classification criteria (including a stable aPL serology at diagnosis) ${ }^{4}$, with a regular followup (at least 1 visit per year) in the same center for at least 15 years starting from diagnosis. Six European referral centers (rheumatology or internal medicine departments) were involved. Patients with a diagnosis of a connective tissue disease (CTD), inflammatory arthritis, or systemic vasculitis (according to the most recent international classification criteria) at the beginning of the followup were excluded. Serological abnormalities, such as positivity for autoantibodies other than aPL and/or complement reduction, were not considered as an exclusion criterion if not associated with clinical manifestations. The study was performed according to the Declaration of Helsinki. Approval was obtained from the Spedali Civili of Brescia ethics committee (protocol no. 1088).

Statistical analysis. Categorical variables were reported as proportion and/or percentage. Continuous variables were reported as mean $( \pm \mathrm{SD})$ or median (range) value. The Fisher's exact test or chi-square test for categorical variables and the Student $t$ test or Wilcoxon-Mann-Whitney test for continuous variables as appropriate were used. $\mathrm{P}$ values $<0.05$ were considered significant. When significant, OR with $95 \% \mathrm{CI}$ was indicated. The probability of being free from recurrences among different groups was calculated using Kaplan-Meier survival curves that were compared by the Breslow test.

\section{RESULTS}

Study cohort. The study included 115 patients with PAPS ( $88 \%$ women and $97 \%$ white), followed between 1983 and 2014 (median followup 18, range 15-30 yrs). Data retrieved from medical charts are reported in Table $1^{4,5}$. The mean $( \pm$ SD) age was $33( \pm 10)$ years at diagnosis. The diagnosis of PAPS had been based on a thrombotic history in 63 patients $(55 \%)$, on obstetrical history in $31(27 \%)$, and on both in $21(18 \%)$. Stroke ( $\mathrm{n}=23,20 \%$ of patients), deep venous thrombosis $(n=43,37 \%)$, and fetal death $(n=39$, $38 \%$ of female patients) were the most common arterial, venous, and obstetrical events at onset, respectively. The serological profiles of the included patients are available in the first column of Table 2. Catastrophic APS (CAPS) was the first manifestation in 2 patients ( 1 in puerperium and 1 following an infection).

After diagnosis, 62 patients (54\%) received oral anticoagulants (AC), 3 in primary prevention based on an obstetrical onset; 32 of the 62 patients (52\%) received oral AC for $<1$ year. Fifty-four patients (47\%) received an antiplatelet drug, 13 of them (24\%) in association with oral AC. Twelve patients $(10 \%)$ did not receive any drug for the longterm prevention of thrombosis at diagnosis, despite in 3 cases a recent thrombotic event (all diagnosed before 2000). Antiplatelet drugs and oral AC were the most prescribed drugs both at diagnosis and during the followup (Table 3). A significant variation in the treatment strategy occurred over the years, including a significantly higher use of hydroxychloroquine (HCQ), other immunosuppressants, and antihypertensive drugs.

Thromboses during followup. Fifty patients (44\%), including 6 with only obstetrical history, experienced 1 or more thrombotic events during a median followup of 18 years, yielding a total of 75 thrombotic events. Sixteen patients had more than 2 thrombotic events during followup (up to 4 thromboses in 2 patients). The mean time to thrombosis during the followup was 104 months (range 0.4-224). The annual incidence of thrombosis was $3.5 \%$ (3\% in patients taking oral $\mathrm{AC}, 5 \%$ in patients not taking oral $\mathrm{AC}$ ). Deep vein thromboses $(n=23)$ and stroke $(n=14)$ were the most frequent venous and arterial events. The site of the first thrombotic event (arterial vs venous) did not influence the site of the first recurrence: venous thrombotic recurrences were more frequent in both groups. In particular, distribution of recurrences was venous in $61 \%$ and arterial in $39 \%$ of patients with venous onset compared with $60 \%$ and $40 \%$, respectively, for patients with arterial onset. A history of arterial events was not associated with an increased rate of thrombotic recurrence ( $47 \%$ in those with positive history vs $42 \%$ in those with negative history, $\mathrm{p}=0.58)$. Six patients $(5 \%)$ developed an episode of CAPS. All of them had triple aPL positivity (compared with 54\% in patients without CAPS, $\mathrm{p}=0.007)$. No patients with CAPS had a history of similar previous events. A trigger was reported in 2 cases that developed CAPS (infection and puerperium).

Treatment regimen at the time of thrombotic recurrences is shown in Figure 1A. A trigger was identified in 18 cases (24\%): 6 infections, 5 puerperium, 3 bridging therapy after surgery, 1 active cancer, and 3 after oral AC withdrawal. In the 84 patients with thrombotic disease onset, the chronic use of oral AC did not significantly reduce the recurrence of thrombosis in the long term (Figure 1B) when compared with other treatment strategies. A new thrombotic event occurred in $49 \%$ of patients currently treated with oral AC and $62 \%$ of those without it (chi-square $p=0.26$ ). The time to first throm-

Personal non-commercial use only. The Journal of Rheumatology Copyright @ 2017. All rights reserved. 
Table 1. List of the items collected from clinical charts.

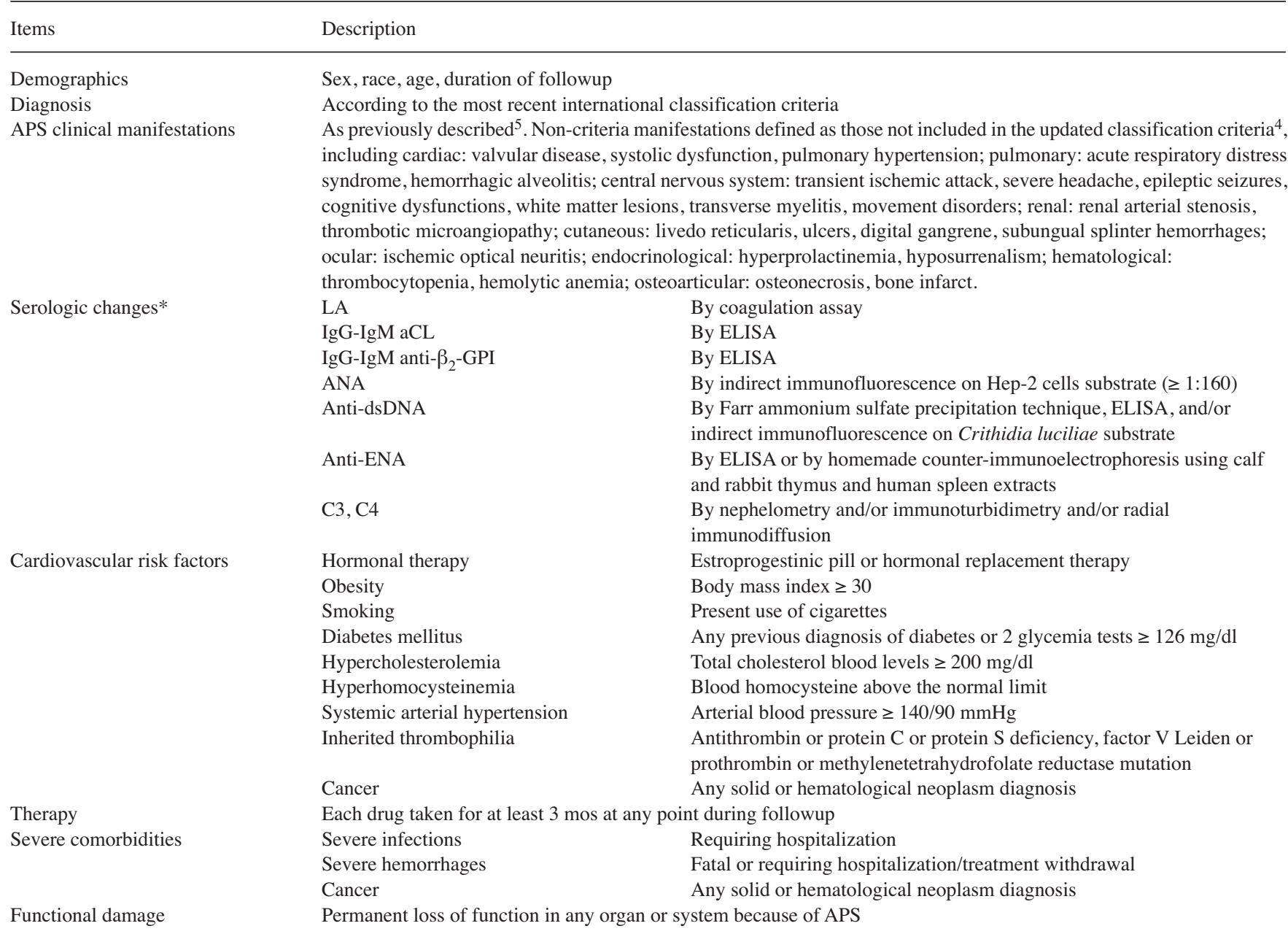

* Only confirmed serological positivity was considered (at least 2 determinations, for antiphospholipid antibodies at least 12 weeks apart). APS: antiphospholipid syndrome; LA: lupus anticoagulant; Ig: immunoglobulin; aCL: anticardiolipin antibodies; anti- $\beta_{2}$-GPI: anti- $\beta_{2}$ glycoprotein I antibodies; ANA: antinuclear antibodies; anti-ENA: antiextractable nuclear antigens antibodies; $\mathrm{C} 3$ and $\mathrm{C} 4$ : complement fractions.

botic recurrence was not significantly different in patients receiving oral $\mathrm{AC}$ versus those not receiving oral $\mathrm{AC}$ (median time 221 vs 198 mos, $p=0.33$ ). Kaplan-Meier survival curves are shown in Figure 2. In patients taking oral AC, a history of arterial events was not associated with an increased thrombotic recurrence rate (33\% in those with positive history vs $31 \%$ in those with negative history, $\mathrm{p}=1.000$ ). Patients who experienced thrombotic events during followup had more frequently a thrombotic disease onset than those who remained free of them $(88 \%$ vs $61 \%$, chi-square $p=0.002$, OR $4.80,95 \%$ CI 1.65-14.7). No other features were significantly associated with thrombotic events during followup.

Pregnancies during followup. Fifty-two women (51\%) had a total of 87 pregnancies during the followup. The outcomes were 5 voluntary abortions (6\%), 6 early miscarriages (7\%), 8 fetal deaths (9\%), and 68 live births (78\%). Complications of live births included 18 preterm deliveries (27\%), 7 hyper- tensive disorders (10\%), and 3 intra-uterine growth restriction (4\%). Excluding the 5 voluntary abortions, 77 of the 82 pregnancies $(94 \%)$ received a prophylactic treatment (low-dose aspirin in $46 \%$, heparin in 31\%, low-dose aspirin plus heparin in $23 \%$ ) variably associated with steroids (34\%), HCQ (6\%), azathioprine (6\%), intravenous immunoglobulins $(\mathrm{Ig} ; 10 \%)$, and plasma exchange $(8 \%)$. The absence of any treatment during pregnancy $(n=5)$ was significantly associated with an adverse outcome, with 3 fetal deaths and 2 miscarriages ( $100 \%$ vs $11 \%$, Fisher's exact test, $p<0.001$, OR infinite, $95 \%$ CI 4.84 to infinite).

APS phenotypes during followup. Fifty-nine patients (51\%) experienced at least 1 new clinical (thrombotic or obstetrical) event attributable to APS according to the classification criteria. Additionally, the distribution of clinical features of APS changed in several patients during followup. In particular, the number of patients with both events (obstet-

Personal non-commercial use only. The Journal of Rheumatology Copyright (c) 2017. All rights reserved. 
Table 2. Serological characteristics and cardiovascular risk factors: comparison between diagnosis and end of followup. Values are $\mathrm{n}(\%)$ unless otherwise specified.

\begin{tabular}{lccc}
\hline Feature & Diagnosis & End of Followup* & p, Chi-square \\
\hline LA positivity & $78 / 107(73)$ & $55 / 85(65)$ & 0.29 \\
aCL-IgG positivity & $103 / 115(90)$ & $89 / 115(77)$ & $\mathbf{0 . 0 2}$ \\
aCL-IgM positivity & $54 / 115(47)$ & $40 / 115(35)$ & 0.08 \\
Anti- $\beta_{2}$-GPI-IgG positivity & $64 / 98(65)$ & $82 / 115(71)$ & 0.43 \\
Anti- $\beta_{2}$-GPI-IgM positivity & $40 / 98(41)$ & $45 / 115(39)$ & 0.91 \\
Single aPL positivity & $22 / 90(24)$ & $10 / 85(12)$ & $\mathbf{0 . 0 3}$ \\
Double aPL positivity & $17 / 90(19)$ & $13 / 85(15)$ & 0.67 \\
Triple aPL positivity & $51 / 90(57)$ & $48 / 85(57)$ & 0.98 \\
Negative aPL & $0 / 90(0)$ & $14 / 85(16)$ & $<\mathbf{0 . 0 0 1}$ \\
ANA positivity & $60 / 115(52)$ & $70 / 115(61)$ & 0.23 \\
Anti-dsDNA positivity & $18 / 115(16)$ & $13 / 115(11)$ & 0.31 \\
Anti-ENA positivity & $12 / 115(10)$ & $20 / 115(17)$ & 0.11 \\
Isolated low C3 & $15 / 108(14)$ & $16 / 111(14)$ & 1.00 \\
Isolated low C4 & $9 / 108(8)$ & $8 / 111(7)$ & 0.95 \\
Low C3 and C4 & $12 / 108(11)$ & $15 / 111(14)$ & 0.74 \\
Hormonal therapy & $23 / 101(23)$ & $2 / 101(2)$ & $<\mathbf{0 . 0 0 1}$ \\
Obesity, BMI $>30$ & $11 / 115(10)$ & $17 / 115(15)$ & 0.31 \\
Smoking & $27 / 115(23)$ & $18 / 115(16)$ & 0.18 \\
Diabetes mellitus & $2 / 115(2)$ & $7 / 115(6)$ & 0.17 \\
Hypercholesterolemia & $26 / 115(23)$ & $41 / 115(36)$ & $\mathbf{0 . 0 4 2}$ \\
Hyperhomocysteinemia & $12 / 85(14)$ & $24 / 113(21)$ & 0.27 \\
Systemic arterial hypertension & $23 / 115(20)$ & $44 / 115(38)$ & $\mathbf{0 . 0 0 4}$ \\
Inherited thrombophilia** & $26 / 103(25)$ & $26 / 103(25)$ & 1.00 \\
Cancer & $1 / 115(1)$ & $8 / 115(7)$ & $\mathbf{0 . 0 4 1}$ \\
\hline
\end{tabular}

* The last 2 determinations (at least 12 weeks apart for aPL) available at the end of the followup were considered. ** Seven patients with heterozygous methylene tetrahydrofolate reductase mutation, 10 with homozygous methylene tetrahydrofolate reductase mutation, 3 with heterozygous factor II mutation, 3 with heterozygous factor $\mathrm{V}$ mutation, 5 with protein $\mathrm{S}$ deficiency, 2 with protein $\mathrm{C}$ deficiency, 2 with antithrombin deficiency. Significant data are in bold face. LA: lupus anticoagulant; aCL: anticardiolipin antibodies; Ig: immunoglobulins; anti- $\beta_{2}$-GPI: anti- $\beta_{2}$ glycoprotein I antibodies; aPL: antiphospholipid antibodies; ANA: antinuclear antibodies; anti-ENA: antiextractable nuclear antigens antibodies; $\mathrm{C} 3$ and $\mathrm{C} 4$ : complement fractions 3 and 4; BMI: body mass index.

Table 3. Treatment at diagnosis and cumulative use during the followup. Values are $\mathrm{n}(\%)$ unless otherwise specified.

\begin{tabular}{lccc}
\hline Therapy & Diagnosis & During Followup & $\mathrm{p}$, Chi-square \\
\hline Corticosteroids* & $34 / 115(30)$ & $49 / 115(43)$ & 0.06 \\
Hydroxychloroquine & $4 / 115(3)$ & $52 / 115(45)$ & $\mathbf{< 0 . 0 0 1}$ \\
Immunosuppressants** & $1 / 115(1)$ & $16 / 115(14)$ & $\mathbf{< 0 . 0 0 1}$ \\
Antiplatelet agents & $54 / 115(47)$ & $68 / 115(59)$ & 0.09 \\
Oral anticoagulant & $62 / 115(54)$ & $77 / 115(67)$ & 0.06 \\
Antihypertensive drugs & $22 / 115(19)$ & $51 / 115(44)$ & $<\mathbf{0 . 0 0 1}$ \\
\hline
\end{tabular}

* Used during pregnancy in most cases. ** Azathioprine, cyclophosphamide, cyclosporine, mycophenolate mofetil, and methotrexate. Significant data are in bold face.

rical and thrombotic) increased: 6 out of 31 patients (19\%) without a history of thrombosis and only pregnancy complications developed a thrombotic event during the followup, and 6 out of 49 female patients (12\%) with a history of thrombosis developed an obstetrical adverse outcome.

Changes of other clinical and biological features of APS during the followup. The most frequent "non-criteria" clinical manifestations observed at diagnosis were cardiac, neurological, cutaneous, and hematological. No significant changes were observed during the followup (data not shown). The comparison of serological features and $\mathrm{CV}$ risk factors at the beginning and at the end of the followup is shown in Table 2. Notably, 15 of 85 patients (18\%) who were tested for all the 3 tests at the end of followup had a completely negative aPL panel. Sixty-seven percent and $71 \%$ of patients had at least 1 traditional $\mathrm{CV}$ risk factor at diagnosis and at the end of the followup, respectively.

Organ damage accrual during followup. At the end of the followup, 34 patients (29\%) had functional organ damage, 5 having more than 1 site of functional damage. This included neurological damage in 18 (cognitive dysfunction/dementia in 13 , aphasia in 2 , ataxia in 2 , hemiparesis in 7 ), chronic heart failure in 7 , significant visual loss in $5(<6 / 10$ visual acuity), chronic renal failure in 4 (creatinine clearance $<60$ $\mathrm{ml} / \mathrm{min}$ ), adrenal insufficiency in 3 , amputation because of a peripheral arterial thrombosis, joint replacement because of an osteonecrosis (without steroid use), and cachexia because of chronic bowel ischemia in 1 each. 
A.

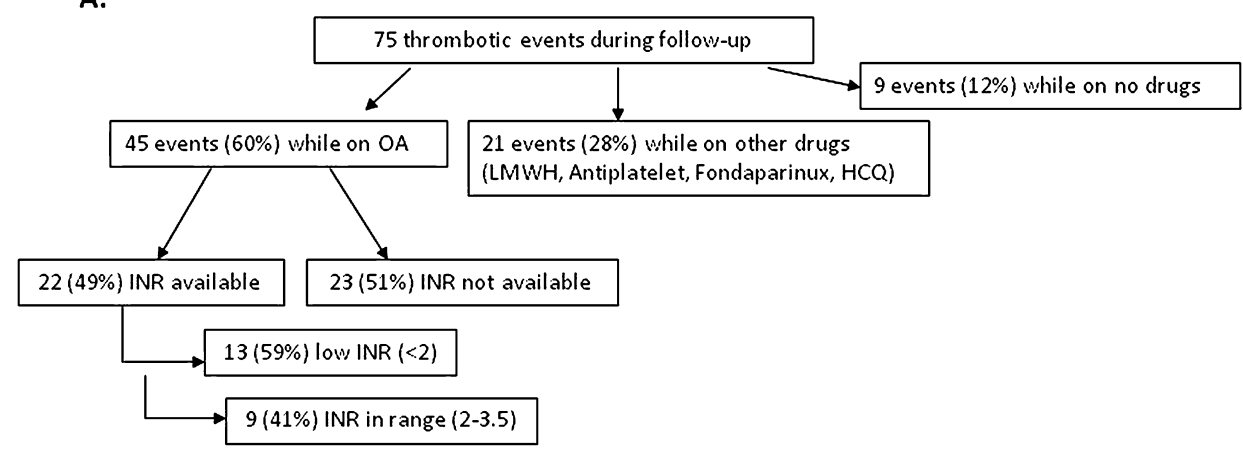

B.

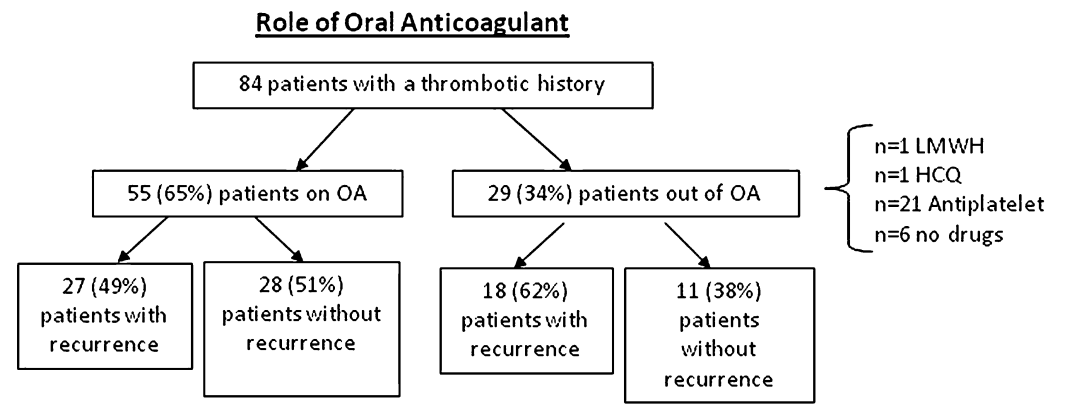

p:0.26

Figure 1. Treatment flow diagram. (A) Treatment at the time of 75 thrombotic events in 51 patients during followup. (B) Involvement of oral anticoagulant in 84 patients with a thrombotic disease onset. OA: oral anticoagulant; INR: international normalized ratio; LMWH: low molecular weight heparin; HCQ: hydroxychloroquine.

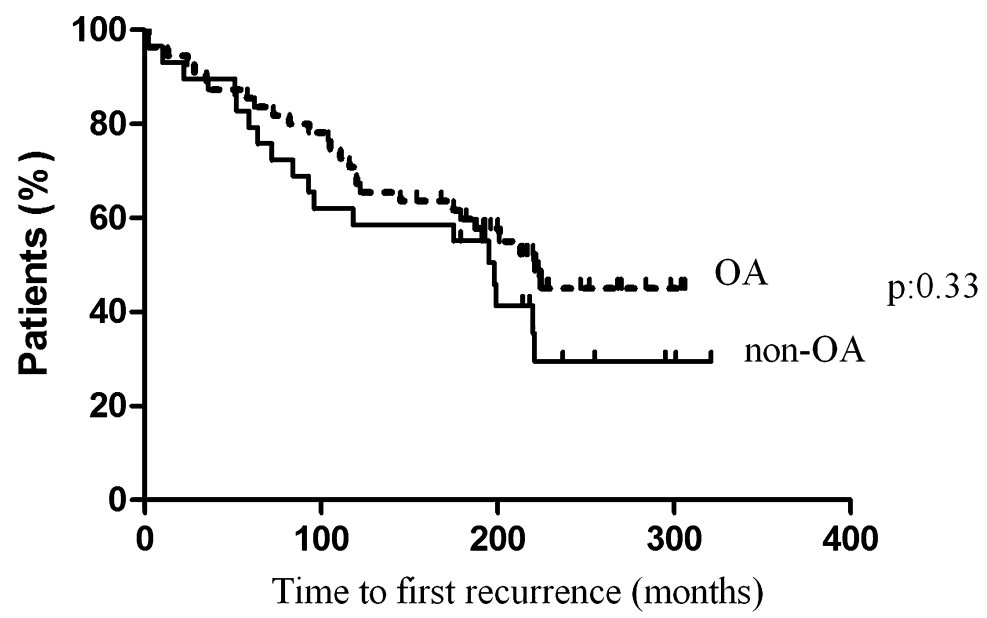

Figure 2. Time (months) to first recurrence in patients with previous thrombosis according to the use and non-use of OA (Kaplan-Meier analysis). OA: oral anticoagulants.

Functional damage was significantly associated with a thrombotic history $(37 \%$ vs $4 \%$, chi-square $\mathrm{p}=0.004$, OR $13.9,95 \%$ CI $1.8-288.4)$, in particular to an arterial event (48\% vs $10 \%$, chi-square $\mathrm{p}<0.001$, OR $7.9,95 \%$ CI $2.7-24.3$ ), especially stroke (53\% vs $20 \%$, chi-square $\mathrm{p}=$
0.001, OR 4.6, 95\% CI 1.76-11.98). We found no significant association between organ damage and demographic, serological variables, or traditional CV risk factors.

Severe comorbidities during the followup. Twenty-four severe bleeding episodes were observed in 18 patients, all

Personal non-commercial use only. The Journal of Rheumatology Copyright (c) 2017. All rights reserved. 
treated with oral AC (23\%), 4 having more than 1 bleeding episode. Genital tract (metrorrhagia) was the most frequent affected site (29\%), followed by cerebral (23\%), gastrointestinal (GI; 17\%), ENT (14\%), musculoskeletal (9\%), intraabdominal $(4 \%)$, and other sites $(9 \%)$. None of the hemorrhagic episodes was fatal. Information about the international normalized ratio (INR) was available in 8 episodes; in 4 cases INR was above 3.5.

Severe infections occurred during followup in 6 patients (5\%), 3 of whom were treated with immunosuppressants: 2 viral (1 herpetic uveitis, 1 cytomegalovirus infection) and 3 bacterial episodes ( 2 pneumonias, 1 infectious colitis with sepsis), and 1 infected retroperitoneal hematoma with sepsis that was followed by CAPS occurrence. The only case of death occurred in a 49-year-old poorly adherent patient, with a disease duration of 22 years and multiorgan involvement, who could not receive full anticoagulation because of recurrent bleeding episodes. The patient died of sepsis originating from a chronically ischemic GI tract.

A diagnosis of cancer was made in 8 patients (7\%): 5 breast, 2 colon, and 1 hepatic at a mean age of 51 years $( \pm 6)$.

Occurrence of other autoimmune diseases during followup. Some patients changed their clinical profile over the years: 7 patients developed systemic lupus erythematosus (SLE), 2 Sjögren syndrome, and 4 undifferentiated CTD after a mean followup period of 110 months ( \pm SD 82). Positivity for antiextractable nuclear antigens antibodies (anti-ENA), especially Ro-SSA, appeared to be a risk factor (38\% vs 6\%, Fisher's exact test $\mathrm{p}=0.003$, OR $10,95 \%$ CI 2.06-49.99) for evolution toward CTD in general. There were no serologic or clinical characteristics associated with evolution toward SLE.

Three patients developed an organ-specific autoimmune disease: 1 multiple sclerosis, 1 primary biliary cirrhosis plus Basedow disease, and 1 autoimmune hepatitis. Overall, 16 patients (14\%) developed another autoimmune disease; 13 of them (11\%) were CTD.

\section{DISCUSSION}

APS is responsible for severe manifestations such as thromboembolic disorders and pregnancy losses occurring often at a young age. While much interest has been devoted to the treatment of the different disease manifestations, limited data are available on the longterm prognosis and few authors have focused on the primary form of APS ${ }^{6,7}$. We had the opportunity to gather data from several tertiary European centers involved in diagnosis and care of PAPS since the 1980s. This enabled us to observe the longterm followup of a number of patients receiving and not receiving the currently recommended treatment and to compare their clinical outcomes. Our population had noteworthy disease severity: $31 \%$ had at least an arterial thrombotic event at onset and 25\% developed at least 1 episode of arterial thrombosis during followup. In contrast to current recommendations ${ }^{8}$, a high proportion of patients discontinued oral $\mathrm{AC}$ in the first year and a minority did not receive any treatment after the thrombotic event, reflecting a lack of knowledge at the time of how best to manage thrombosis in patients with APS.

About half of the cohort experienced at least 1 new clinical event attributable to APS during the followup. We recorded 75 thrombotic events during the followup, 6 in patients with only pregnancy complications, without a history of thrombosis. The overall thrombotic rate in our cohort (44\%) was higher than that found by Cervera, et al $(31 \%)^{3}$, but lower than those reported in the $90 \mathrm{~s}^{9,10,11,12}$. The site of the initial thrombosis (arterial vs venous) was not predictive of the site of the recurrence as reported in some studies ${ }^{13,14}$. The history of previous clots was associated with thrombosis recurrence, a finding in contrast with other cohorts of shorter followup ${ }^{15,16}$. In our study, despite the limitation in retrieving the effective intensity of anticoagulation, the chronic use of oral AC did not appear to be protective. This could possibly reflect the problems linked to the use of oral AC in real life for a long time (e.g., patient's adherence to treatment, drugs' interactions, etc.) that may influence its effectiveness ${ }^{17}$; the frequent finding of a low INR at the time of recurrence may support this hypothesis. However, recurrences were also observed in patients with an INR in the recommended range, emphasizing that thrombosis can recur when the drug should be effective.

A high prevalence of other $\mathrm{CV}$ risk factors was observed in our population, but they did not influence the overall frequency of thrombosis during followup in this group of treated patients. Inherited thrombophilia was represented in one-fourth of patients, underlining the risk of interaction with aPL in the pathogenesis of thrombotic events.

Regarding treatment, we observed that at the end of the followup, $67 \%$ of patients were taking oral $\mathrm{AC}$, as expected based on the high rate of thrombotic history $(78 \%)$ and the current treatment recommendations. We also noticed a higher tendency in using immunomodulatory drugs, perhaps attributable to the development of clinical manifestations of CTD and with the emerging evidence of the potential benefits of HCQ.

The incidence of CAPS during the followup (5\%) was higher than similar previous studies would suggest ${ }^{3,18}$. The rate of survival after CAPS was higher than expected, but possibly explained by our exclusion criteria, because patients who died before completing the 15 years of followup were not entered into our study.

The gestational outcome of pregnancies occurring during the followup and therefore under prophylaxis was significantly improved compared with baseline, with a high chance of live births ${ }^{3,6,9}$. However, the risk of prematurity remained high. This emphasizes the need for dedicated management of these high-risk pregnancies because the currently recommended treatment does not completely reverse the harm of the disease ${ }^{19}$.

Personal non-commercial use only. The Journal of Rheumatology Copyright $\odot$ 2017. All rights reserved. 
Organ damage accrual is not a well-defined concept in APS because the Systemic Lupus International Collaborating Clinics Damage Index seems inadequate and the recently proposed APS-specific index is still under validation ${ }^{20}$. We found that $29 \%$ of patients developed functional damage, and neurologic damage was the most frequent, as previously observed $^{2}$. A history of arterial thrombosis, in particular stroke, was the only statistically significant risk factor for organ damage accrual. Similar findings were also reported in 2 retrospective cohort ${ }^{2,6}$. In 1 study $^{6}$, organ damage was shown in one-third of patients, while one-fifth had functional impairment. The other study ${ }^{2}$ reported damage in $29 \%$ of patients (45 with APS associated to SLE), but the definition of organ damage was broader, including manifestations not necessarily associated with permanent loss of function.

Increased morbidity in APS may be related to causes other than APS itself. In particular, antithrombotic treatment increased the risk of severe bleeding episodes. This complication occurred in $23 \%$ of patients, all receiving oral AC. The rates reported in the literature vary from $3 \%$ to $23 \% \%^{7,11,13,9}$, but comparisons are difficult because drug regimens and definitions of severity differ widely. The musculocutaneous system was the most frequent site of bleeding in the Europhospholipid cohort ${ }^{3}$, in contrast to our cohort in which the genital tract was most frequently involved. Cerebral hemorrhage was observed in some of our patients, as in other studies $^{3}$. Severe infections were rarely recorded in our patients, but were the cause of death in 1 patient with chronic ischemic bowel disease because of arterial stenosis. Other studies have reported bacterial infections as the leading nonthrombotic cause of death in $\mathrm{APS}^{3}$. An association between aPL and malignancies, in particular hematological, has been well documented ${ }^{21}$, and cancer is one of the most frequent causes of death in patients with $\mathrm{APS}^{3}$. In our cohort, neoplasms were diagnosed in a minority of patients, but they were all solid and the majority reflected the 2 most common histological findings reported in the general European population (breast and colon carcinoma) ${ }^{22}$.

One aim of our study was to investigate whether clinical features at presentation remain stable or change over time; we found no significant variation in prevalence of non-criteria clinical manifestations. Cardiac, central nervous system, cutaneous, and hematological involvement were the most represented, according to the Europhospholipid Cohort ${ }^{23}$.

Among aPL, anticardiolipin antibodies-IgG were the most frequent antibodies similar to other cohorts ${ }^{2,3,6,8,12}$. Triple aPL positivity was the most frequent profile, underlining the full-blown APS picture of the patients included in our cohort. The aPL positivity tended to decline during the followup. The low rate of tested anti- $\beta_{2}$-GPI at diagnosis and of lupus anticoagulant at the end of the followup must be related to the delayed introduction of this test and to the concomitant use of oral AC, respectively. Different autoantibody speci- ficities, apart from aPL, have been described in PAPS. Antinuclear antibodies (ANA) ${ }^{24}$, anti-ENA (anti-Ro/SSA in most of cases) ${ }^{25}$, and anti-dsDNA ${ }^{26}$ can be positive in a minority of patients. In addition, antinucleosome antibodies have been described in patients with PAPS ${ }^{27}$. Some authors suggest that the presence of autoantibodies other than aPL, particularly anti-dsDNA by Farr test or anti-ENA, should be included in the exclusion criteria for a PAPS diagnosis ${ }^{28}$. In our paper, only patients with full-blown autoimmune clinical features were excluded because a real-life PAPS patient with or without autoantibodies basically receives the same management. In our cohort, ANA was confirmed as the most frequent positivity, but their presence was not associated with an increased risk for thrombosis, as reported in another study ${ }^{29}$. Only $16 \%$ of anti-dsDNA positivity was present at diagnosis without any associated clinical manifestations while anti-ENA (mainly anti-Ro/SSA), found in $10 \%$ of patients, were associated to the occurrence of CTD. Reduced levels of complement were present in a minority of patients, as previously described ${ }^{7}$. Evolution toward a CTD was observed in a number of patients (11\%), with SLE being the most frequent. The mean time for PAPS to develop a CTD was 9 years, in line with what was reported in another study $^{30}$.

Other groups ${ }^{7,30}$ have reported a similar rate of evolution even though their followup periods were shorter.

Our study has several limitations: the relatively small sample size, the retrospective design, the lack of a centralized laboratory, and the lack of INR availability in many patients at the time of events. However, it should be considered that PAPS is a relatively uncommon disease and patients with a longterm followup are even more uncommon. It is important to note that patients were regularly followed in the same referral center for over several years and each center had its own laboratory testing for aPL with validated and comparable methods $^{31}$. The requirement of a long followup as selection method and the involvement only of referral centers may have contributed to selection bias in that only patients with more severe manifestations of PAPS were included. In addition, it is possible that the comparison of aPL results between disease onset and the end of the followup could be biased by the evolution of laboratory techniques over the years.

Nonetheless, our study has a number of strengths. Our study provides information on the possible longterm disease evolution of patients presenting only with PAPS, as well as the effectiveness of currently recommended treatments over time. It is also noteworthy that our patients were followed in the same center for their entire disease course by medical teams with experience in APS, which enables a greater chance of consistency in data collection. The multicenter design may also provide the advantage of a more representative picture of the disease. Finally, our study is, to our knowledge, the longest followup of patients presenting solely with features of PAPS.

$$
\text { Personal non-commercial use only. The Journal of Rheumatology Copyright () 2017. All rights reserved. }
$$


The results of our longterm study of patients with PAPS suggest a relatively poor prognosis with a high risk of functional damage and morbidity. Further, efforts should be made to identify more effective strategies to prevent thrombotic recurrences and organ damage so that the quality of life and survival of these young patients may be improved.

\section{ACKNOWLEDGMENT}

The authors thank Rajesh Kumar for support with statistical analysis.

\section{REFERENCES}

1. Hughes GR. The anticardiolipin syndrome. Clin Exp Rheum 1985;3:285-6.

2. Grika EP, Ziakas PD, Zintzaras E, Moutsopoulos HM, Vlachoyiannopoulos PG. Morbidity, mortality, and organ damage in patients with antiphospholipid syndrome. J Rheumatol 2012;39:516-23.

3. Cervera R, Serrano R, Pons-Estel GJ, Ceberio-Hualde L, Shoenfeld Y, de Ramón E, et al; Euro-Phospholipid Project Group (European Forum on Antiphospholipid Antibodies). Morbidity and mortality in the antiphospholipid syndrome during a 10-year period: a multicentre prospective study of 1000 patients. Ann Rheum Dis 2015;74:1011-8

4. Miyakis S, Lockshin MD, Atsumi T, Branch DW, Brey RL, Cervera $\mathrm{R}$, et al. International consensus statement on an update of the classification criteria for definite antiphospholipid syndrome (APS). J Thromb Haemost 2006;4:295-306.

5. Taraborelli M, Andreoli L, Tincani A. Much more than thrombosis and pregnancy loss: the antiphospholipid syndrome as a 'systemic disease'. Best Pract Res Clin Rheumatol 2012;26:79-90.

6. Erkan D, Yazici Y, Sobel R, Lockshin MD. Primary antiphospholipid syndrome: functional outcome after 10 years. J Rheumatol 2000;27:2817-21.

7. Gómez-Puerta JA, Martín H, Amigo MC, Aguirre MA, Camps MT, Cuadrado MJ, et al. Long-term follow-up in 128 patients with primary antiphospholipid syndrome: do they develop lupus? Medicine 2005;84:225-30

8. Ruiz-Irastorza G, Cuadrado MJ, Ruiz-Arruza I, Brey R, Crowther M, Derksen R, et al. Evidence-based recommendations for the prevention and long-term management of thrombosis in antiphospholipid antibody-positive patients: report of a task force at the 13th International Congress on antiphospholipid antibodies. Lupus 2011;20:206-18.

9. Muñoz-Rodriguez FJ, Font J, Cervera R, Reverter JC, Tàssies D, Espinosa G, et al. Clinical study and follow-up of 100 patients with the antiphospholipid syndrome. Semin Arthritis Rheum 1999;29:182-90.

10. Krnic-Barrie S, O'Connor CR, Looney SW, Pierangeli SS, Harris EN. A retrospective review of 61 patients with antiphospholipid syndrome. Analysis of factors influencing recurrent thrombosis. Arch Intern Med 1997:157:2101-8.

11. Khamashta MA, Cuadrado MJ, Mujic F, Taub NA, Hunt BJ, Hughes GR. The management of thrombosis in the antiphospholipid-antibody syndrome. N Engl J Med 1995; 332:993-7.

12. Rosove MH, Brewer PM. Antiphospholipid thrombosis: clinical course after the first thrombotic event in 70 patients. Ann Intern Med 1992;117:303-8.

13. García-Carrasco M, Galarza C, Gómez-Ponce M, Cervera R, Rojas-Rodríguez J, Espinosa G, et al. Antiphospholipid syndrome in Latin American patients: clinical and immunologic characteristics and comparison with European patients. Lupus 2007;16:366-73.

14. Meroni PL, Borghi MO, Raschi E, Tedesco F. Pathogenesis of antiphospholipid syndrome: understanding the antibodies. Nat Rev Rheumatol 2011;7:330-9.

15. Hernández-Molina G, Espericueta-Arriola G, Cabral AR. The role of lupus anticoagulant and triple marker positivity as risk factors for rethrombosis in patients with primary antiphospholipid syndrome. Clin Exp Rheumatol 2013;31:382-8.

16. Bazzan M, Vaccarino A, Stella S, Sciascia S, Montaruli B, Bertero MT, et al. Patients with antiphosholipid syndrome and thrombotic recurrences: a real world observation (the Piedmont cohort study). Lupus 2016;25:479-85.

17. Palareti G, Antonucci E, Lip GY, Testa S, Guazzaloca G, Falanga A, et al; START-Register Participants. The SAME-TT2R2 score predicts the quality of anticoagulation control in patients with acute VTE. A real-life inception cohort study. Thromb Haemost 2016;115:1101-8.

18. Cervera R; CAPS Registry Project Group. Catastrophic antiphospholipid syndrome (CAPS): update from the 'CAPS Registry'. Lupus 2010;19:412-8.

19. Andreoli L, Bertsias GK, Agmon-Levin N, Brown S, Cervera R, Costedoat-Chalumeau N, et al. EULAR recommendations for women's health and the management of family planning, assisted reproduction, pregnancy and menopause in patients with systemic lupus erythematosus and/or antiphospholipid syndrome. Ann Rheum Dis 2017;76:476-85.

20. Alba P, Gómez-Puerta JA, Goycochea-Robles MV, Amigo MC. Organ damage and quality of life in antiphospholipid syndrome. Curr Rheumatol Rep 2016;18:7.

21. Tincani A, Taraborelli M, Cattaneo R. Antiphospholipid antibodies and malignancies. Autoimmun Rev 2010;9:200-2.

22. Ferlay J, Parkin DM, Steliarova-Foucher E. Estimates of cancer incidence and mortality in Europe in 2008. Eur J Cancer 2010;46:765-81.

23. Cervera R, Piette JC, Font J, Khamashta MA, Shoenfeld Y, Camps MT, et al; Euro-Phospholipid Project Group. Antiphospholipid syndrome: clinical and immunologic manifestations and patterns of disease expression in a cohort of 1,000 patients. Arthritis Rheum 2002;46:1019-27.

24. Asherson RA, Baguley E, Pal C, Hughes GR. Antiphospholipid syndrome: five year follow up. Ann Rheum Dis 1991;50:805-10.

25. Ceribelli A, Tincani A, Cavazzana I, Franceschini F, Cattaneo R, Pauley BA, et al. Anti-argonaute2 (Ago2/Su) and -Ro antibodies identified by immunoprecipitation in primary anti-phospholipid syndrome (PAPS). Autoimmunity 2011;44:90-7.

26. Smeenk RJ, Lucassen WA, Swaak TJ. Is anticardiolipin activity a cross-reaction of anti-DNA or a separate entity? Arthritis Rheum 1987;30:607-17.

27. Andreoli L, Pregnolato F, Burlingame RW, Allegri F, Rizzini S, Fanelli V, et al. Antinucleosome antibodies in primary antiphospholipid syndrome: a hint at systemic autoimmunity? J Autoimmun 2008;30:51-7.

28. Piette JC, Wechsler B, Frances C, Papo T, Godeau P. Exclusion criteria for primary antiphospholipid syndrome. J Rheumatol 1993;20:1802-4.

29. de Carvalho JF, Caleiro MT, Vendramini M, Bonfá E. Clinical and laboratory evaluation of patients with primary antiphospholipid syndrome according to the frequency of antinuclear antibodies (ANA Hep-2). Rev Bras Reumatol 2010;50:262-72.

30. Freire PV, Watanabe E, dos Santos NR, Bueno C, Bonfá E, de Carvalho JF. Distinct antibody profile: a clue to primary antiphospholipid syndrome evolving into systemic lupus erythematosus? Clin Rheumatol 2014;33:349-53.

31. Tincani A, Allegri F, Balestrieri G, Reber G, Sanmarco M, Meroni P, et al. Minimal requirements for antiphospholipid antibodies ELISAs proposed by the European Forum on antiphospholipid antibodies. Thromb Res 2004;114:553-8. 